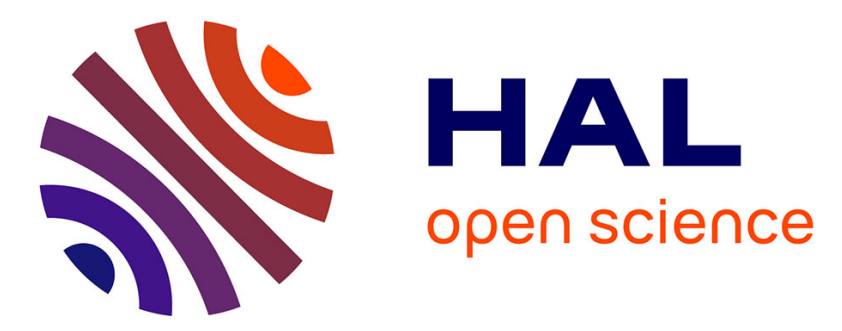

\title{
Entropy as a measure of variability and stemness in single-cell transcriptomics
}

Olivier Gandrillon, Mathilde Gaillard, Thibault Espinasse, Nicolas B Garnier, Charles Dussiau, Olivier Kosmider, Pierre Sujobert

\section{To cite this version:}

Olivier Gandrillon, Mathilde Gaillard, Thibault Espinasse, Nicolas B Garnier, Charles Dussiau, et al.. Entropy as a measure of variability and stemness in single-cell transcriptomics. Current Opinion in Systems Biology, 2021, 27, pp.1-14. 10.1016/j.coisb.2021.05.009 . hal-03378505

\section{HAL Id: hal-03378505 https://hal.science/hal-03378505}

Submitted on 14 Oct 2021

HAL is a multi-disciplinary open access archive for the deposit and dissemination of scientific research documents, whether they are published or not. The documents may come from teaching and research institutions in France or abroad, or from public or private research centers.
L'archive ouverte pluridisciplinaire $\mathbf{H A L}$, est destinée au dépôt et à la diffusion de documents scientifiques de niveau recherche, publiés ou non, émanant des établissements d'enseignement et de recherche français ou étrangers, des laboratoires publics ou privés. 


\section{Entropy as a measure of variability and stemness in single-cell transcriptomics}

Olivier Gandrillon ${ }^{1,2,7}$, Mathilde Gaillard ${ }^{1,3}$, Thibault Espinasse ${ }^{2,3}$, Nicolas B. Garnier ${ }^{4}$, Charles Dussiau ${ }^{5}$, Olivier Kosmider ${ }^{5}$, and Pierre Sujobert ${ }^{6}$

${ }^{1}$ Univ Lyon, ENS de Lyon, Univ Claude Bernard, CNRS UMR 5239, INSERM U1210, Laboratory of Biology and Modelling of the Cell, 46 allée d'Italie Site Jacques Monod, F-69007, Lyon, France

${ }^{2}$ Inria Team Dracula, Inria Center Grenoble Rhône-Alpes, France

${ }^{3}$ Univ Lyon, Université Lyon 1, CNRS UMR5208, Institut Camille Jordan, 43 blvd du

11 novembre 1918, F-69622 Villeurbanne-Cedex, France

${ }^{4}$ Univ Lyon, Ens de Lyon, Univ Claude Bernard, CNRS UMR 5672, Laboratoire de

Physique, F-69342 Lyon, France

${ }^{5}$ Université de Paris, Institut Cochin, CNRS UMR8104, INSERM U1016, Département

Développement, Reproduction, Cancer, Paris, France. Assistance Publique-Hôpitaux

de Paris. Centre - Université de Paris, Service d'Hématologie biologique, Hôpital

Cochin, Paris, France.

${ }^{6}$ Service d'Hématologie Biologique - Hospices Civils de Lyon - 165, Chemin du Grand

Revoyet, 69495, Pierre-Bénite Cedex

${ }^{7}$ Corresponding author: Olivier.Gandrillon@ens-lyon.fr

14 octobre 2021

\section{Why use entropy?}

Biology can be described as evolving between typological and population thinking, as proposed by Ernst Mayr [12]. In other words, biology might either focus on the type (i.e. the invariant or Plato's eidos), or focus on the population (in evolutionary biology), or the variation away from the type. Charles Darwin is of course the main contributor of this new way of thinking, where variation comes first. Statistics have evidently taken the main stage when it came to quantify the extent of variability, through the concept of variance. More recently concepts from physical statistics, like entropy, have been tentatively used as a metrics for variability. Initially forged for measuring the disorder or uncertainty in a physical system, then reused within the frame of information theory, it is now being increasingly used as a metrics for variability and used as a proxy for stemness in single cell transcriptomics data. The goal of that paper is to show that the same metrics can be used to measure very different things and to clarify for the biologist what is captured by entropy depending on its various usage.

There has been an occasional use of entropy for population-based measurements (see e.g. [22]; [2]) but the recent explosion of single-cell based measurements has really led to a strong increase in the use of entropy as a relevant metric for quantifying variability.

One of the first paper to advocate for the use of entropy as a measure of variability was [39]. The authors suggested that "This connection suggests a broad principle : at equilibrium, cell populations that are subject to strict regulatory constraints should exhibit well-defined and low entropy expression patterns, whereas those that are subject to weaker regulatory constraints should exhibit more diverse, higher entropy expression patterns. Viewing variability in this light indicates that PSC (pluripotent stem cells) populations may be more diverse than differentiated populations because they are subject to weaker regulatory constraints". The authors went on to propose an information-theoretic interpretation of stem cell dynamics that views cellular multipotency as an instance of maximum entropy statistical inference [51].

Since this first proposal, there has been a vast amount of literature that made use of entropy for the analysis of single cell transcriptomics data. Those types of data have to be seen as of distributions of 
values [40], due to the unavoidable stochastic nature of gene expression [34]. This massive use led to a situation where the use of the term "entropy" can be misleading, because it measures different things.

We therefore propose in the present paper a simple typology that should help non-specialists readers to better grasp the relevance of that term, and differentiate its various occurences.

\section{What is entropy?}

Entropy was first introduced by Rudolf Clausius to express the degradation of energy in thermodynamics at the time of the first industrial revolution [21]. Ludwig Boltzmann later revealed that this thermodynamical entropy could indeed be expressed as a measure of uncertainty, or mixed-up-ness of a physical system, i.e., a measure of the unpredictability of the microscopic degrees of freedom that are unknown to an observer recording macroscopic variables [5]. In the 1940's, this measure of disorder was generalized by Claude E. Shannon to form the basis of a new scientific field : "Information theory" [14,61]. Within this perspective, any signal, measurement or random variable can be assigned an entropy which measures its unpredictability, or in more celebrated terms its information contents.

Entropy is a functional of the probability density function (PDF). This simply means that given a signal $X$, with a probability density function $p(x)$ which is nothing but the normalized histogram of $X$, the entropy can be computed using a formula that only involves $p$ :

$$
H(X)=-\int_{S} p(x) \log (p(x)) d x .
$$

In this expression, we have assumed that $X$ takes continuous values, e.g. real numbers, and we have noted $S$ the support of the PDF, i.e., the set of all possible values that $X$ can take. In that case, $p$ is said to be continuous and the entropy is often referred to as the differential entropy. Another important class of signals correspond to those which take discrete values, e.g. integer values. In that case, $p$ is said to be discrete and the entropy is expressed by the famous Shannon formula

$$
H(X)=-\sum_{m \in S} p(m) \log (p(m)) .
$$

In practice, and in particular when dealing with single cell transcriptomics, it is usually more comfortable to use discrete distributions. This is natural not only if data is composed of integers (for example a number of molecules), but it is also the form that appears when constructing the histogram of $X$ to estimate $\mathrm{p}$, whether it is discrete or continuous. The histogram is composed of successive bins indexed by an integer $m \in[1 ; M]$, where $M$ is the number of bins in the histogram (see section 2 below). Each bin represents an interval of possible values for $X$, and $p(m)$ is related to the probability for the variable $X$ to have its value in the bin $m$.

Beware that the Shannon entropy computed after this binning procedure does not estimate consistently the differential entropy. Therefore, one has to be careful when comparing entropies of distributions using this binning step.

In practice, it is possible [72] to compute instead $-\sum_{m \in S} \hat{p}(m) \log \left(\frac{\hat{p}(m)}{w(m)}\right)$, where $w(m)$ corresponds to the length of the $m^{\text {th }}$ bin and $\hat{p}(m)$ correspond to an estimation of $p(m)$ (for instance the proportion of the sample that falls in bin $m$ ).

Other authors (e.g. [32]) proposed to use a normalized entropy, for instance by rescaling Shannon entropy with its maximum value $\log (M):-\frac{1}{\log (M)} \sum_{m \in S} \hat{p}(m) \log (\hat{p}(m))$ to get a quantity between 0 and 1.

The entropy as defined by Shannon is a measure of the uncertainty or information contained in a signal. If the probability is uniform (think about a regular dice with 6 perfectly equi-probable values, $p(m)=p=1 / 6$ for all $m \in[1 . .6])$, then the uncertainty is maximal, and so is the entropy. On the contrary, if one bin or one of the possible outcome has much larger probability (think about a loaded dice), than the uncertainty is smaller, and so is the entropy (see Figure 1). 


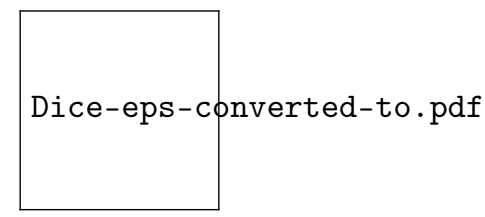

Figure 1 - In the case of a regular dice, the entropy is equal to $-\left(1 / 6 \times \log _{2}(1 / 6)\right) \times 6$, that is 2.58 . In the case of the loaded dice the entropy is equal to $-\left(1 / 2 \times \log _{2}(1 / 2)\right) \times 2$, that is 1 .

The extreme case where only a single value has a non-vanishing probability — which is then equal to 1 - corresponds to a Dirac distribution (see Glossary below), which has an entropy equal to 0, according to the Shannon formula.

Additionally, the differential entropy evolves as the logarithm of the variance of the distribution : if one considers the reduced variable $y=(x-\mu) / \sigma$, where $\bar{x}$ denotes the expected value of $X$ and $\sigma$ its standard deviation, then

$$
H(X)=H(Y)+\log \sigma,
$$

which indicates that the entropy of a dataset $X$ increases with its standard deviation ${ }^{1}$.

Entropy is therefore a measure of the uncertainty, understood as the possible surprise of an outcome, and hence a measure of the information contained in the probability distribution. One part of the entropy is due to the standard deviation of the signal, while another part is related to the shape of its distribution. As such, it offers a fully relevant measure of variability [9].

\section{How to compute entropy}

In this review we will focus on the use of entropy for analyzing single-cell omics data. Those are information regarding the molecular content of one cell at a time [77]. We will more specifically focus on single-cell transcriptomics data, where a number of mRNA molecules for different genes is determined at the single-cell level.

There are a number of open issues regarding the proper handling of such single-cell mRNA expression values. It ranges from the definition of a proper statistical model to account for the specific nature of those data, including a high proportion of null values ( [11], [55]), up to normalization issues [13]. We refer the interested reader to a recent review [38].

Single-cell mRNA expression has been shown to be well fitted using a Gamma distribution ( [1] ; see Glossary below). It has been shown that a negative binomial distribution, the discrete version of the Gamma law can be derived under certain conditions (i.e. in a bursty regime) from a mathematical analysis of a two-states model for gene expression [60]. If one assumes that this is a correct model for the data, and that the data are good enough for a proper estimation of the parameters of the Gamma distribution, then one can derive the entropy from the analytical expression of the Gamma distribution ${ }^{2}$.

The alternative path consists in a non parametric estimations of the entropy, as follows.

A single-cell omics experiment will generate values, for example Ct values in the case of an RTqPCR experiment or the number of mRNAs detected into a number of cells in the case of an RNA-seq experiment. From such continuous or discrete data, one must first estimate the probability distribution the values were drawn from in order to calculate the entropy. Although this might seems from a biologist's point of view like a trivial task, especially in the discrete case of RNA-seq data, a closer examination shows that this is a really difficult question (see e.g [37], [28], [47]). It can be rephrased as how good is the estimate the entropy $H(X)$ given by :

$$
\hat{H}(X)=-\sum_{m \in M} \hat{p}_{m} \log \left(\hat{p}_{m}\right)
$$

where $\hat{p}_{m}$ is the (normalized) frequency at which one can find cells or genes values in the given bin indexed by $m$. One should note here that this value is computer for one cell, or one gene (see below), in other term that it is a univariate distribution. No multivariate version of entropy has been proposed for

1. The entropy of $X$ can be understood as the sum of two terms : the entropy of $Y$ (the normalized version of $X$, which is independent of $\sigma$ ) which relates to the shape of the PDF, and $\log \sigma$ which describes the width of the PDF.

2. $H=\alpha-\ln (\beta)+\ln \Gamma(\alpha)+(1-\alpha) \psi(\alpha)$ with $\alpha=\mu^{2} / \sigma$ and $\beta=\mu / \sigma, \Gamma$ being the Gamma function and $\psi$ being the digamma function. 
the single cell field, although it might help to better characterize datasets of intrinsically multivariate nature.

$\hat{p}_{m}$ is not the real probability but an estimate of that probability based upon a limited data set, and on the choice of the bins. The question then arises as to what is the best binning procedure, and how it impacts the resulting calculation.

Let's take an example. Say we have measured the height of 5 persons :

\begin{tabular}{cc}
\hline Person & height $(\mathrm{m})$ \\
\hline P1 & 1.56 \\
P2 & 1.70 \\
P3 & 1.85 \\
P4 & 1.66 \\
P5 & 1.78
\end{tabular}

One can now decide to regroup those individuals by the following size class (bin) :

\begin{tabular}{ccc}
\hline bin & count & pdf \\
\hline$[1.5 ; 1.6[$ & 1 & $1 / 5$ \\
{$[1.6 ; 1.7[$} & 1 & $1 / 5$ \\
{$[1.7 ; 1.8[$} & 2 & $2 / 5$ \\
{$[1.8 ; 1.9[$} & 1 & $1 / 5$ \\
\hline
\end{tabular}

The data is then partitioned in discrete boxes (bins) and in each of these one can calculate $\hat{p}_{m}$, and thus deduce the estimate $\hat{H}=7 / 5 \log 5-4 / 5 \log 2=1.699$. But one can also decide to use a more compact view of the original dataset. This can simply be achieved by increasing the size of the bins, e.g. from 0.1 to 0.2 , to get :

\begin{tabular}{ccc}
\hline bin & count & pdf \\
\hline$[1.5 ; 1.7[$ & 2 & $2 / 5$ \\
{$[1.7 ; 1.9[$} & 3 & $3 / 5$ \\
\hline
\end{tabular}

which leads to an estimate $\hat{H}=4 / 5 \log (2 / 5)+9 / 5 \log (3 / 5)=1.653$, that is smaller than the former one.

At that stage, it is worth mentioning that the maximum likelihood estimation $\hat{H}(X)$ of Shannon entropy is always negatively biased [3] :

$$
\mathbb{E}_{p}[\hat{H}(X)] \leq H(p)
$$

This means that natural estimation of entropy exhibits a tendency to underestimation. Figure 2 illustrates this property. However this bias vanishes as the number of observations increases [3]. 


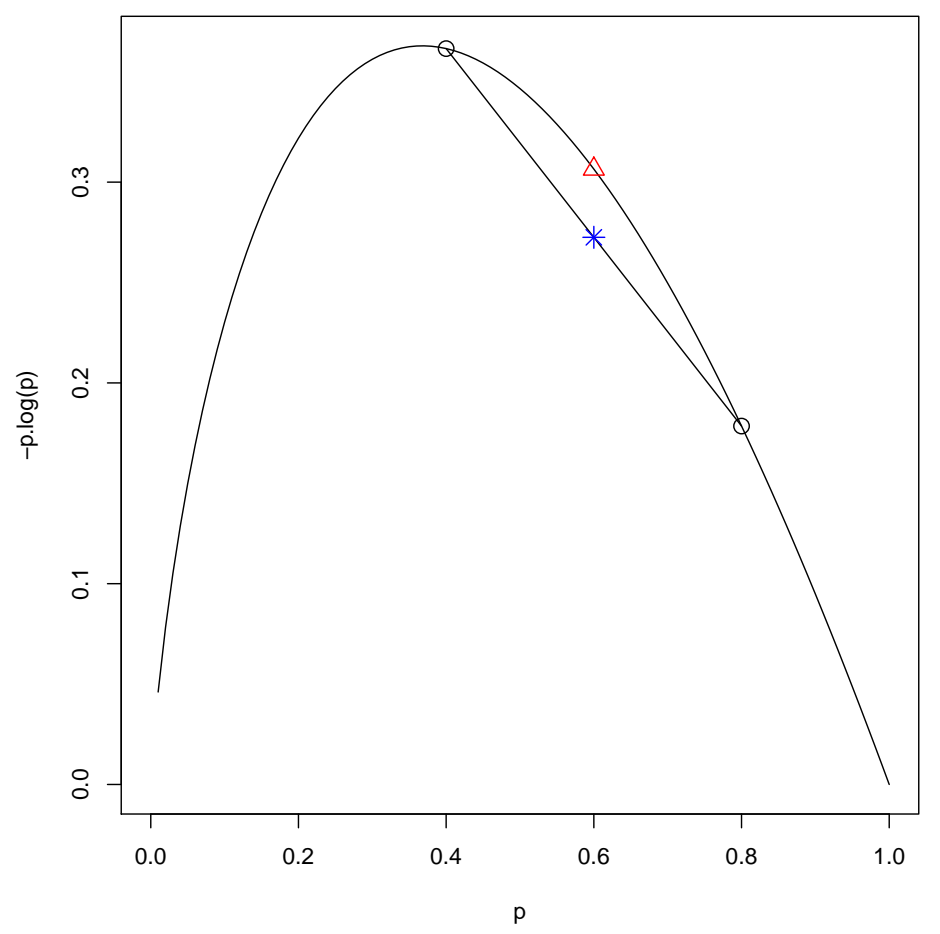

Figure 2 - Illustration of the bias in entropy estimation. The function $g: p \mapsto-p \log (p)$ is mapped here. For any random variable $\hat{p}$, we have, $\mathbb{E}[g(\hat{p})] \leq g(\mathbb{E}[\hat{p}])$ thanks to the concavity of $g$. For instance, if $\hat{p}$ takes values $0.4,0.8$ (black dots) with probability $1 / 2$, then $\mathbb{E}[\hat{p}]=p_{0}=0.6$ and $\mathbb{E}[g(\hat{p})]$ is given by the $y$-coordinate of the blue dot, whereas $g\left(p_{0}\right)$ is given by the $y$-coordinate of the red triangle. Hence, $\mathbb{E}[-\hat{p} \log (\hat{p})] \leq-p_{0} \log \left(p_{0}\right)$. By summing over all bins, this illustrates why $\mathbb{E}[\hat{H}(X)]=-\sum_{i} \mathbb{E}\left[\hat{p}_{i} \log \left(\hat{p}_{i}\right)\right] \leq-\sum_{i} p_{i} \log \left(p_{i}\right)=H(p)$.

There is an abundant litterature as to what should be the proper way of defining a relevant bining size. It has been shown that extreme bin size (either too small or too large) introduce a bias in the estimation [49]. Sophisticated methods have been proposed for a proper bin size estimate, ranging from the use of Doane's rule ( [48]; [16]), an extension of Sturges' formula, to the use of the Bayesian Blocks algorithm, a method designed to find an optimal binning for a set of values without enforcing uniform bin width $([66] ;[56])$. The very existence of so many methods shows how the proper binning still is an open and difficult question.

Such a binning issue is relevant for scRTqPCR data where the initial values are expressed as a function of the $\mathrm{Ct}$ which is a continuous value. It is also relevant in the case of discrete values like the counting of molecules in scRNAseq experiments : one can choose a number of bins that is equal to the highest number of molecules detected in any cell of the dataset (and hence a bin size equal to 1), or increase the bin size up to having only two bins : one for the zero expression level and the other one for any non-zero expression level [76].

\section{When to study entropy?}

We would first like to point out that one can calculate two sorts of entropies : an IntRAcellular Entropy and an IntERcellular Entropy (Figure 3). The IntRAcellular Entropy is endowing a CELL with an entropy value, whereas the IntERcellular Entropy is endowing a GENE with an entropy value. In other words, in the IntRAcellular Entropy, one try to capture the heterogeneity of the transcriptional state of a given cell. In the IntERcellular Entropy, one try to capture the heterogeneity of the transcriptional state of a given gene across a population of cells.

Based upon a thorough literature search we can then refine this proposal and classify entropy for single cell omics data analysis into four different categories : one can estimate an entropy per cell or 


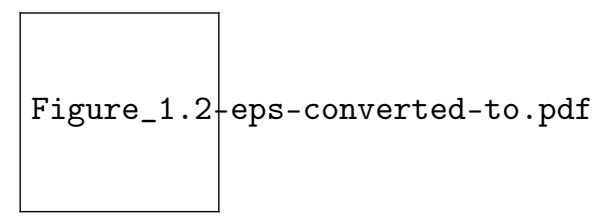

Figure 3 - A schematic description of the two different entropies. On the first line are displayed two cells with their mRNA content for three genes. The second line shows how one can estimate an IntRAcellular Entropy. Here the entropy for Cell 1 is equal to $-\left(1 / 3 \times \log _{2}(1 / 3)\right) \times 3$, that is 1.5 . The entropy for Cell 2 is equal to $-\left(\left(1 / 9 \times \log _{2}(1 / 9)\right)+\left(3 / 9 \times \log _{2}(3 / 9)\right)+\left(5 / 9 \times \log _{2}(5 / 9)\right)\right)$, that is 1.3 . The mean IntRAcellular entropy is therefore of 1.4. The third line shows how one can estimate an IntERcellular Entropy. Here the entropy for Gene B is null, and the entropy for both Gene A and C is equal to $-\left(1 / 2 \times \log _{2}(1 / 2)\right)$, that is 0.5 . The mean IntERcellular Entropy is therefore of 0.33

an entropy per gene, and one can use external information (like Gene Ontology of a protein-potein interaction network) to compute this entropy or obtain it directly from a given distribution. All the available literature can be classified in such a way (Table 1 below).

\begin{tabular}{|l|l|l|}
\hline & IntRAcellular Entropy & IntERcellular Entropy \\
\hline \multirow{3}{*}{ Requires external information } & SLICE [27], scEntropy [36], & \\
& MCE [63], SCENT [68], & \\
& scEntropy [78] & \\
\hline \multirow{5}{*}{ Without external information } & dpath [24], StemId [25], & \\
& single-cell entropy [36], & Shannon entropy [18], [50], \\
& Shannon entropy [48], & [66], [76] \\
& Palantir [59],CEE [73], scEn- & \\
& tropy [78], scRCMF, [79] & \\
\hline
\end{tabular}

TABLE 1 - The four entry table for entropy use in single-cell omics. In the case of [78] and [36], the entropy calculation is based upon a Reference Cell (see below) which can be either an external input of based upon an intrinsic calculation from the dataset. There is no know example of an IntERcellular Entropy requiring external information, but it could be easily designed, for example by using a given gene expression distribution as a reference.

\subsection{IntRAcellular Entropy requiring an external information}

In the SLICE algorithm [27], Gene Ontology (GO) clusters define the external information. Genes are first attributed to those GO clusters, and then the algorithm computes the entropy of the clusters distributions. A high entropy is linked with a flat distribution where all GO clusters are more or less equally represented (i.e. contains a similar number of genes), and there are many potential functions harboured by the cells, whereas a reduced entropy is linked with a distribution that concentrates on a smaller amount of clusters/functions, being the hallmark of a differentiated cell.

For the single-cell entropy (SCENT) algorithm, the external information comes from a proteinprotein interaction (PPI) network [68]. scRNA-Seq values are superimposed on top of this PPI network. Once more, immature stem cells are defined by more promiscuous signaling pathways, whereas more mature cells are characterized by a narrower distribution. This was an extension of an entropy measure that was initially defined over bulk tissue data [6].

A similar philosophy was introduced in [63] with the Markov Chain Entropy (MCE) where the authors compute a Markov transition matrix (see Glossary below) through a PPI. They demonstrate that the MCE behavior is driven by a correlation between mRNA expression and network connectivity, and conclude that it outperforms three other single-cell potency models.

\subsection{IntRAcellular Entropy without an external information}

The StemId algorithm [25] computes a cell-based entropy $H=-\sum_{i, j} p_{i, j} \log \left(p_{i, j}\right)$ where $p_{i, j}=$ $n_{i, j} / N$ and $n_{i, j}$ equals the number of transcripts of gene $\mathrm{i}$ in cell $\mathrm{j} . \mathrm{N}$ equals the total number of transcripts in each cell, which has been normalized. From this the authors compute an entropy score for 
each cluster, as defined by k-medoids clustering, in order to identify stem cell clusters [25]. The authors assume that a more promiscuous transcriptome, reflected in a higher entropy, would be expected for stem cells, when a more confined transcriptome would be expected for a mature cell type.

The Palantir algorithm [59] computes yet another entropy value, that also requires the construction of a Markov transition matrix (see Glossary) with branches. In contrast with MCE, this transition is built from the data, and not from an external information. One can then compute a vector of branch probabilities to reach each of the possible terminal states, and compute the entropy of such a vector. It allows to define a differentiation potential in the sense that immature cells have a higher probability to reach a large number of terminal states. This indicator, as the previous ones, decrease monotonously as differentiation proceeds.

The dpath algorithm computes a metagene entropy [24]. It is based upon the construction of metagenes (vectors of genes) using a weighted Poisson non-negative matrix factorization (wp-NMF) method, and on the computation of an entropy on the metagene coefficients V, a probabilistic simplex that indicated the relative weight of each metagene in each cell. The metagene entropy of cell $\mathrm{m}$ is then defined as $-\sum_{k=1}^{K} V_{k m} \log \left(V_{k m}\right)$. This metagene entropy serves as a measure of how many distinct programmes (parts) are active (expressed) in a cell and was significantly higher in progenitor cells compared with more differentiated cells. It therefore allowed the ranking of cells based on their differentiation potential.

Up to that point all observations concluded toward a monotonous decrease of IntRAcellular Entropy as a function of the differentiation process.

The only exception concern the study of the very early development in human and mouse ( [36], [48], [79]). In the first two studies, the authors observe a steady increase from the oocyte up to the blastocyst stage ( [36], [48]). They propose that such an increase in heterogeneity would have been necessary for cell fate diversifications [48]. Note that the second exception uses the single-cell entropy (scEntropy) metric, where $p_{i}(y)$ is the distribution density of the components $y_{i, j}$ in $y_{i}=x_{i}-r, \mathrm{r}$ being the gene expression vector $\mathrm{r}$ of a Reference Cell [36]. The third study, using the scRCMF algorithm which is a very similar NMF approach as [24] describes a transient increase of entropy during what the author describe as "transition states" [79]. The authors do not discuss the discrepancy between their results and the previously published ones.

The Cellular Entropy Estimator [73] is defined as $-\sum_{j=1}^{K} P_{i, j} \log \left(P_{i, j}\right)$ where $\left(P_{i, j}\right)$ is the probability for the cell $\mathrm{i}$ to be attributed to the cluster $\mathrm{j}$ by the SoptSC algorithm [74]. As such it is more a measure of the ability of a cell to transition to a new state than a measure of stemness per se.

Up to this point, most of the studies have shown that the very early development shows a monotonous increase in cellular entropy, whereas differentiation from adult stem cells shows a monotonous decrease in entropy. The only two exception are the use of scEntropy metrics which was shown to increase during the differentiation of iPSCs into cardiomyocytes [78] and the use of scRCMF during mammalian preimplantation development [79]. In the first case, it could be the choice of the Reference Cell that influences such a directionality of entropy changes.In the second case, it would be interesting to see the result of the application of dpath [24], a very similar algorithm, to an early mammalian development dataset.

\subsection{IntERcellular Entropy without an external information}

In any case, the IntRAcellular entropy shows mostly a monotonous behaviour. This is in sharp contrast with the study by [50]. In this work, we proposed an IntERcellular version of Shanon entropy, computed from the raw data. For each gene, one can compute its entropy from its distribution in a population of cells. By measuring single cell gene expression during the differentiation of chicken erythroid progenitors, we demonstrated the existence of a surge in variability in gene expression in the midst of this erythroid differentiation process, with a final differentiated state reaching a lower entropy that the initial one. This was the first example of a differentiation process where IntERcellular Entropy could evolve in a non-monotonous way.

Very similar results have been obtained in a different setting [66] : the authors have been measuring single cell expression by RTqPCR during the differentiation of mouse embryonic stem cells along the neuronal lineage. Using a very similar version of Shanon entropy as in [50], they also observed an increase in variability at the beginning of the differentiation process.

Such a non-monotonous behaviour of entropy was further corroborated by [76]. To solve the binning issue (see upper), the authors argue that only two obviously separated levels can be easily distinguished : the zero expression level and the greater-than-zero expression level. This led them to propose a binary Shannon entropy, calculated on two bins. Although this might seem like a very different measure than [50] or [66], they also demonstrate the existence of a surge in entropy in long-term haematopoietic stem cell differentiation as well as in EML cell line erythroid commitment. 
More recently, we computed a gene-based Shannon entropy on Single-cell RNA-seq data from normal and pathological human bone marrow. We demonstrated the surge in entropy in the main hematopoietic differentiation pathways on normal and myelodysplastic syndromes samples. We also showed that entropy is increased in hematopoietic stem cell of myelodysplastic syndromes as compared to age matched control, suggesting a role for gene expression variability in the pathophysiology of this disease [18].

One key point in computing an IntERcellular Entropy lies within the proper definition of the group of cells on which the gene entropy is computed. In [50] and [66] the cells have been harvested at different time points offering a natural cell grouping scheme. In [76] the authors group the cells by predefined cell types, based upon known gene expression patterns. In [18] we propose a more data-driven approach : the cells are first ordered by their pseudo-time as assessed by Slingshot [64], and the entropy is calculated on sliding windows across such a pseudo-time. Various re-ordering scheme do exists ( [57], [7]; [8]), but they have not yet been systematically assessed for their ability to reorder cells in an entropy-relevant manner.

\subsection{Why IntRAcellular and IntERcellular entropy differ?}

At that stage it is remarkable that almost all techniques aiming at measuring an IntRAcellular entropy do show a monotonous decrease in entropy as the differentiation proceeds, whereas all IntERcellular metrics point toward a bell-shaped non-monotonous behavior.

To propose an explanation for those observation, we would like to make a reasoning on a toy dataset, represented in Tables 2 and 3. We display in those matrices six cells, ranging from stem to mature, and 4 genes. The stem cells are characterized by a low and promiscuous expression of all the genes, thereby preserving their potential for different lineages choices. As differentiation proceeds, some genes get repressed and some get activated. This tends to concentrate the gene expression pattern on a smaller number of genes, thereby reducing the entropy. Ultimately, the mature cell type is characterized by the expression of a small number of genes (here one), and a low entropy.

In order to account for the surge in IntERcellular entropy, one should now focus on the intermediate "progenitor" population. In this case, one propose that cells are passing through a stage of so called 'hesitant' behaviour [44]. During this stage, each cell explores (at its own pace and independently of cell division) many different possibilities before reaching a stable combination of genes to be expressed. This peak of uncertainty can be captured by entropy measurements (see Table 3), but also through the reconstruction of transcriptional uncertainty landscapes [23], or through the mathematical definition of a potential energy of a population of cells [70]

\begin{tabular}{|l|c|c|c|c|c|}
\hline & Gene 1 & Gene 2 & Gene 3 & Gene 4 & Entropy \\
\hline Cell 1 & 1 & 2 & 4 & 3 & 2 (High) \\
Cell 2 & 1 & 2 & 3 & 4 & 2 (High) \\
Cell 3 & 5 & 7 & 0 & 0 & 1.5 (Medium) \\
Cell 4 & 0 & 0 & 8 & 5 & 1.5 (Medium) \\
Cell 5 & 15 & 0 & 0 & 0 & 0.8 (Low) \\
Cell 6 & 18 & 0 & 0 & 0 & 0.8 (Low) \\
\hline
\end{tabular}

TABLE 2 - Monotonous decrease in cell-based entropy. Cells 1 and 2 are stem cells, 3 and 4 are progenitor cells and 5 and 6 are mature cells characterized by the elevated expression of Gene 1 . All the distribution gets progressively concentrated on one gene.

\section{Discussion}

It is no wonder why the entropy concept has become so popular in the era of single cell omics. Indeed, even though the stochastic nature of gene expression has been anticipated for quite some times ( [31], [33]), single cell studies have established its unavoidable nature ( [34], [20], [67]). This led to the critical need for tools that can help to make sense of distribution-based evidence [40] away from the classical mean-based vision [34].

Although all the example in this review are from the single-cell transcriptomics field, one should note that most, if not all, of the known molecular techniques are being adapted to single cell studies [77]. The resultant high-dimensional single cell data generated require new theoretical approaches and analytical algorithms for effective visualization and interpretation. Statistical physics tools and especially 


\begin{tabular}{|c|c|c|c|c|c|}
\hline & Gene 1 & Gene 2 & Gene 3 & Gene 4 & Mean entropy \\
\hline Cell 1 & 1 & 2 & 4 & 3 & \\
Cell 2 & 1 & 2 & 3 & 4 & \\
Ent & 0 & 0 & 1 & 1 & 0.5 (Low) \\
\hline Cell 3 & 5 & 7 & 0 & 0 & \\
Cell 4 & 0 & 0 & 8 & 5 & \\
Ent & 1 & 1 & 1 & 1 & 1 (High) \\
\hline Cell 5 & 15 & 0 & 0 & 0 & \\
Cell 6 & 18 & 0 & 0 & 0 & 0.25 (Low) \\
Ent & 1 & 0 & 0 & 0 & 0 \\
\hline
\end{tabular}

TABLE 3 - Non-monotonous behavior of gene-based entropy. Cells 1 and 2 are stem cells, 3 and 4 are progenitor cells and 5 and 6 are mature cells characterized by the expression of Gene 1. The intermediary phase is characterized by a state of uncertainty in which entropy is peaking ( [50], [44], [23])

information theoretic ones, like entropy, therefore became largely used. There are of course more uses of entropy in biology, both within (see e.g. [35]) and outside of the single-cell omics field (see e.g. [30], [13]), but that would be beyond the scope of this review to analyze them all.

Beyond the need for a consensual binning procedure (see upper), one currently missing tool is the absence of a statistical test for comparing the entropy of two distributions and to decide whether or not their entropy is significantly different. We are currently working on this issue.

Other indicators of variability do exist, from the variance, up to the coefficient of variation $(\mathrm{CV}$; $\sigma / \mu)$, the Fano factor $\left(\sigma^{2} / \mu\right)$ and the normalized variance $\left(\sigma^{2} / \mu^{2}\right)$. Some authors have proposed a measure of "transcriptional noise" based on pairwise cell-cell distanced calculated as $d=\sqrt{(1-\rho) / 2}$, with $\rho$ being the Spearman's rank correlation coefficient [42].

One has to state here that entropy stands out from those measures, since it can be shown to measure the variability of the elements within a given distribution, and that its expression is not arbitrary, as it is the only linear indicator for such a concept [9].

All measures of molecular variability are influenced by a variety of potentially confounding factors, including a gene's mean expression [19]. Some author proposed to condition CV values on mean by computing the residuals of a non-parametric loess regression of CV-mean [69] to eliminate such a mean dependency.

Such bias advocate for care to be taken when drawing inferences about the role of biological variability using such indicators. Nevertheless, our review shows that the use of entropy was instrumental in highlighting the connection between stemness and uncertainty ( [51]) or between differentiation and an increase in molecular variability ( [26], [43], [17]), leading to the concept of noise-induced differentiation ( [54], [71], [29], [10]).

Altogether, those results strongly support the view that cell differentiation is a probabilistic process ( [33], [46]). This is rooted in the fact that cells are neither machines ( [45]), nor simple information processing devices. Cells (like all living systems) are rooted within a physico-chemical world to which they belong. Their specific complexity nevertheless sometimes led to the idea that they should be treated differently that classical physico-chemical systems ( [58]). But their nature of dynamical systems is exemplified here by the relevance of the use of a statistical physics concept, like entropy.

From a more biological perspective and especially in the field of cancer, further single cells studiesbased entropy analysis of splice variants [41] may help us to better understand the consequences of splicing alteration in the disease establishment and progression as previously suggested in bulk analysis [52]. Moreover, future single cell analysis could consider some poorly explored players of the cells, such as miRNAs [75] or lncRNAs [15] which could vary significantly during normal and pathological processes of differentiation. Lastly, all these considerations have to be explored again in approaches including the consequences of spatial cell-cell interactions ( [62], [65], [53]) for example between hematological stem cells and their niche [4].

In conclusion, we hope the reader is now convinced of the versatility and power of entropy to quantify the extent of variability contained in a distribution, and that next time he/she will investigate how to unravel the importance of variability during a biological process, he/she will not hesitate to make the best use of entropy as a relevant metrics. 


\section{Glossary}

The Dirac delta can be loosely thought of as a function on the real line which is zero everywhere except at the origin, where it is infinite,

$$
\delta(x)=\left\{\begin{array}{c}
+\infty \text { if } x=0 \\
0 \text { if } x \neq 0
\end{array}\right.
$$

and which is also constrained to satisfy the identity

$$
\int_{-\infty}^{\infty} \delta(x) \mathrm{d} x=1 .
$$

Intuitively, it correspond to the distribution of a constant random variable. Such random variable can not be described properly by a probability density function.

The Gamma distribution is a two-parameter family of continuous probability distributions for positive real values. It is highly versatile and it occur frequently in models used in engineering, business or biology for which the variables are always positive and the results are skewed (unbalanced), and therefore not captured by the more "classical" normal distribution.

A Markov transition matrix describes how a random system evolves as a function of time. Formally, a transition matrix $P$ is a real nonnegative square matrix with each row summing to 1 . It number of rows is equal to the number of possible states for the system, and the $i^{\text {th }}$ row is the probability distribution of the state at time $t+1$, knowing it is in state $i$ at time $t$. Hence, $P_{i j}$ corresponds to the probability for the system to be in state $j$ at time $t+1$ knowing it is in state $i$ at time $t$.

Let's imagine three cells, and assume they can transition according to the scheme in Figure 4. Note that the system has to enjoy the so called "Markov property" meaning that once the current state of the system is known, his past does not affect the probability for future states

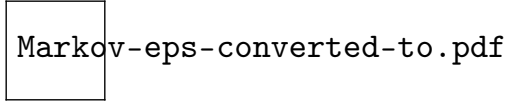

FigURE 4 - Transition graph between three cells

One can then start building $P$ the transition matrix. If one start in state 1 , then one has $1 / 3$ chances to stay in $1,1 / 3$ chances to transition to 2 and $1 / 3$ chances to transition to 3 . This therefore provides us with the first line in $P$ :

$$
P=\left(\begin{array}{lll}
1 / 3 & 1 / 3 & 1 / 3
\end{array}\right)
$$

By repeating the operation when starting from state 2 , and then 3 , one can then complete the matrix :

$$
P=\left(\begin{array}{ccc}
1 / 3 & 1 / 3 & 1 / 3 \\
0 & 1 / 5 & 4 / 5 \\
0 & 0 & 1
\end{array}\right)
$$

One can then compute an entropy per row, and one can see in the example that this entropy will be decreasing from cell $1(H=1.58)$ to cell $3(H=0)$.

\section{Acknowledgments}

This work has been supported by grants from the Rhône-Alpes Complex Systems Institute (IXXI) and from Institut Convergence PLASCAN (ANR-17-CONV-0002). We also thank the BioSyL Federation and the LabEx Ecofect (ANR-11-LABX-0048) of the University of Lyon for inspiring scientific events. We warmly thank Gaël Yvert and Franck Picard for critical reading of the manuscript.

\section{Declaration of interest}

Declaration of interest : none 


\section{$9 \quad$ Highlighted papers}

* of special interest

[20] Laid the foundation for the study of gene expression variability at the single cell level using a two reporter system in E. Coli level.

[1] A technical tour-de-force : counting mRNA and proteins from the same gene at the single cell

[25] The first use of IntRAcellular entropy to characterize stemness at the single cell level.

[10] Demonstrates a functional connection between transcriptome fluctuations andcell decision making

** of outstanding interest

[50] The first demonstration of a non-monotonous behavior of IntERcellular entropy during a differentiation sequence.

[34] The first demonstration of cell-to-cell variability in human cells.

[55] A very thoughtful and relevant analysis of the statistical nature of scRNAseq data

[66] A statistical mechanics view of a differentiation process

\section{Références}

[1] C. Albayrak, C. A. Jordi, C. Zechner, J. Lin, C. A. Bichsel, M. Khammash, and S. Tay. Digital quantification of proteins and mRNA in single mammalian cells. Mol Cell, 61(6) :914-24, 2016.

[2] L. Anavy, M. Levin, S. Khair, N. Nakanishi, S. L. Fernandez-Valverde, B. M. Degnan, and I. Yanai. BLIND ordering of large-scale transcriptomic developmental timecourses. Development, $141(5): 1161-6,2014$.

[3] A. Antos and I. Kontoyiannis. Convergence properties of functional estimates for discrete distributions. Random Structures $\&$ Algorithms, 19(3-4) :163-193, 2001.

[4] C. Baccin, J. Al-Sabah, L. Velten, P. M. Helbling, F. Grunschlager, P. Hernandez-Malmierca, C. Nombela-Arrieta, L. M. Steinmetz, A. Trumpp, and S. Haas. Combined single-cell and spatial transcriptomics reveal the molecular, cellular and spatial bone marrow niche organization. Nat Cell Biol, 22(1) :38-48, 2020.

[5] R. Balian. From Microphysics to Macrophysics. Springer-Verlag, 1991.

[6] C. R. Banerji, D. Miranda-Saavedra, S. Severini, M. Widschwendter, T. Enver, J. X. Zhou, and A. E. Teschendorff. Cellular network entropy as the energy potential in Waddington's differentiation landscape. Sci Rep, $3: 1-7,2013$.

[7] V. Bergen, M. Lange, S. Peidli, F. A. Wolf, and F. J. Theis. Generalizing RNA velocity to transient cell states through dynamical modeling. bioRxiv, page 820936, 2019.

[8] J. Cao, M. Spielmann, X. Qiu, X. Huang, D. M. Ibrahim, A. J. Hill, F. Zhang, S. Mundlos, L. Christiansen, F. J. Steemers, C. Trapnell, and J. Shendure. The single-cell transcriptional landscape of mammalian organogenesis. Nature, 566(7745) :496-502, 2019.

[9] G. Carcassi, C.A. Aidala, and J. Barbour. Variability as a better characterization of shannon entropy. arXiv, 2019.

[10] H. H. Chang, M. Hemberg, M. Barahona, D. E. Ingber, and S. Huang. Transcriptome-wide noise controls lineage choice in mammalian progenitor cells. Nature, 453(7194) :544-7, 2008.

[11] K. Choi, Y. Chen, D. A. Skelly, G Churchill, and A. Bayesian model selection reveals biological origins of zero inflation in single-cell transcriptomics. bioRxiv, page 2020.03.03.974808, 2020.

[12] C. Chung. On the origin of the typological/population distinction in ernst mayr's changing views of species, 1942-1959. Studies in History and Philosophy of Science Part C : Studies in History and Philosophy of Biological and Biomedical Sciences, 34(2) :277 - 296, 2003.

[13] M. B. Cole, D. Risso, A. Wagner, D. DeTomaso, J. Ngai, E. Purdom, S. Dudoit, and N. Yosef. Performance assessment and selection of normalization procedures for single-cell RNA-seq. Cell Syst, 8(4) :315-328 e8, 2019.

[14] T. Cover and Thomas J. Elements of Information Theory. 2nd ed. Wiley, 2006.

[15] Y. Deng, H. Luo, Z. Yang, and L. Liu. Lncas2cancer : a comprehensive database for alternative splicing of lncrnas across human cancers. Brief Bioinform, 2020. 
[16] D.P. Doane. Aesthetic frequency classifications. The American Statistician, 30(4) :181-183, 1976.

[17] A. F. Domingues, R. Kulkarni, G. Giotopoulos, S. Gupta, L. Vinnenberg, L. Arede, E. Foerner, M. Khalili, R. R. Adao, A. Johns, S. Tan, K. Zeka, B. J. Huntly, S. Prabakaran, and C. Pina. Loss of kat2a enhances transcriptional noise and depletes acute myeloid leukemia stem-like cells. Elife, $9,2020$.

[18] C. Dussiau. Tbd. bioRxiv, 2020.

[19] N. Eling, M. D. Morgan, and J. C. Marioni. Challenges in measuring and understanding biological noise. Nat Rev Genet, 20(9) :536-548, 2019.

[20] M. B. Elowitz, A. J. Levine, E. D. Siggia, and P. S. Swain. Stochastic gene expression in a single cell. Science, 297(5584) :1183-1186, 2002.

[21] E. Fermi. Thermodynamics. Dover, 1956.

[22] S. Fuhrman, M.J. Cunningham, X. Wen, G. Zweiger, J.J. Seilhamer, and R. Somogyi. The application of Shannon entropy in the identification of putative drug targets. Biosystems, $55: 5-14$, 2000.

[23] N. P. Gao, O. Gandrillon, A. Páldi, U. Herbach, and R. Gunawan. Universality of cell differentiation trajectories revealed by a reconstruction of transcriptional uncertainty landscapes from single-cell transcriptomic data. bioRxiv, page 2020.04.23.056069, 2020.

[24] W. Gong, T. L. Rasmussen, B. N. Singh, N. Koyano-Nakagawa, W. Pan, and D. J. Garry. Dpath software reveals hierarchical haemato-endothelial lineages of Etv2 progenitors based on single-cell transcriptome analysis. Nat Commun, 8 :14362, 2017.

[25] D. Grun, M. J. Muraro, J. C. Boisset, K. Wiebrands, A. Lyubimova, G. Dharmadhikari, M. van den Born, J. van Es, E. Jansen, H. Clevers, E. J. de Koning, and A. van Oudenaarden. De novo prediction of stem cell identity using single-cell transcriptome data. Cell Stem Cell, 19(2) :266-77, 2016.

[26] A. Guillemin, R. Duchesne, F. Crauste, S. Gonin-Giraud, and O. Gandrillon. Drugs modulating stochastic gene expression affect the erythroid differentiation process. PLOS ONE, 14(11) :e0225166, 2019.

[27] M. Guo, E. L. Bao, M. Wagner, J. A. Whitsett, and Y. Xu. SLICE : determining cell differentiation and lineage based on single cell entropy. Nucleic Acids Res, 45(7) :e54, 2017.

[28] J. Hausser and K. Strimmer. Entropy inference and the James-Stein estimator, with application to nonlinear gene association networks. Journal of Machine Learning Research, 10 :1469-1484, 2009.

[29] M. Hoffmann, H. H. Chang, S. Huang, D. E. Ingber, M. Loeffler, and J. Galle. Noise-driven stem cell and progenitor population dynamics. PLoS ONE, 3(8) :e2922, 2008.

[30] G. Jenkinson, E. Pujadas, J. Goutsias, and A. P. Feinberg. Potential energy landscapes identify the information-theoretic nature of the epigenome. Nat Genet, 49(5) :719-729, 2017.

[31] M.S. Ko. A stochastic model for gene induction. J. Theor. Biol., 153 :181-94, 1991.

[32] U. Kumar, V. Kumar, and J N Kapur. Normalized measures of entropy. International Journal Of General System, 12(1):55-69, 1986.

[33] J.J Kupiec. A probabilistic theory for cell differentiation, embryonic mortality and DNA C-value paradox. Speculations in Science and Technology, 6(5) :471-478, 1983.

[34] J. M. Levsky and R. H. Singer. Gene expression and the myth of the average cell. Trends Cell Biol., 13(1) :4-6, 2003.

[35] B. Liu, C. Li, Z. Li, D. Wang, X. Ren, and Z. Zhang. An entropy-based metric for assessing the purity of single cell populations. Nat Commun, 11(1) :3155, 2020.

[36] J. Liu, Y. Song, and J. Lei. Single-cell entropy to quantify the cellular order parameter from single-cell RNA-seq data. Biophysical Reviews and Letters, $15: 35-49,2020$.

[37] W. M. Lord, J. Sun, and E. M. Bollt. Geometric k-nearest neighbor estimation of entropy and mutual information. Chaos, 28(3) :033114, 2018.

[38] M. D. Luecken and F. J. Theis. Current best practices in single-cell RNA-seq analysis : a tutorial. Mol Syst Biol, 15(6) :e8746, 2019.

[39] B. D. MacArthur and I. R. Lemischka. Statistical mechanics of pluripotency. Cell, 154(3) :484-9, 2013.

[40] J. C. Mar. The rise of the distributions : why non-normality is important for understanding the transcriptome and beyond. Biophys Rev., page 89-94, 2019. 
[41] G. K. Marinov, B. A. Williams, K. McCue, G. P. Schroth, J. Gertz, R. M. Myers, and B. J. Wold. From single-cell to cell-pool transcriptomes : stochasticity in gene expression and RNA splicing. Genome Res, 24(3) :496-510, 2014.

[42] H. Mohammed, I. Hernando-Herraez, A. Savino, A. Scialdone, I. Macaulay, C. Mulas, T. Chandra, T. Voet, W. Dean, J. Nichols, J. C. Marioni, and W. Reik. Single-cell landscape of transcriptional heterogeneity and cell fate decisions during mouse early gastrulation. Cell Rep, 20(5) :1215-1228, 2017.

[43] N. Moris, S. Edri, D. Seyres, R. Kulkarni, A. F. Domingues, T. Balayo, M. Frontini, and C. Pina. Histone acetyltransferase KAT2A stabilizes pluripotency with control of transcriptional heterogeneity. Stem Cells, 2018.

[44] A. Moussy, J. Cosette, R. Parmentier, C. da Silva, G. Corre, A. Richard, O. Gandrillon, D. Stockholm, and A. Paldi. Integrated time-lapse and single-cell transcription studies highlight the variable and dynamic nature of human hematopoietic cell fate commitment. PLoS Biol, 15(7) :e2001867, 2017.

[45] D. J. Nicholson. Is the cell really a machine? J Theor Biol, 477 :108-126, 2019.

[46] A. Paldi. Stochastic or deterministic? That is the question. Organisms. Journal of Biological Sciences, 4 :77-79, 2020.

[47] L. Paninski. Estimation of entropy and mutual information. Neural Computation, 15 :1191-1253, 2003.

[48] V. Piras, M. Tomita, and K. Selvarajoo. Transcriptome-wide variability in single embryonic development cells. Sci Rep, $4: 1-9,2014$.

[49] S. Purwani, J. Nahar, and C. Twining. Analyzing bin-width effect on the computed entropy. AIP Conference Proceedings, 1868(1) :040008, 2017.

[50] A. Richard, L. Boullu, U. Herbach, A. Bonnafoux, V. Morin, E. Vallin, A. Guillemin, N. Papili Gao, R. Gunawan, J. Cosette, O. Arnaud, J. J. Kupiec, T. Espinasse, S. Gonin-Giraud, and O. Gandrillon. Single-cell-based analysis highlights a surge in cell-to-cell molecular variability preceding irreversible commitment in a differentiation process. PLoS Biol, 14(12) :e1002585, 2016.

[51] S. J. Ridden, H. H. Chang, K. C. Zygalakis, and B. D. MacArthur. Entropy, ergodicity, and stem cell multipotency. Phys Rev Lett, 115(20) :208103, 2015.

[52] W. Ritchie, S. Granjeaud, D. Puthier, and D. Gautheret. Entropy measures quantify global splicing disorders in cancer. PLoS Comput Biol, 4(3) :e1000011, 2008.

[53] S. G. Rodriques, R. R. Stickels, A. Goeva, C. A. Martin, E. Murray, C. R. Vanderburg, J. Welch, L. M. Chen, F. Chen, and E. Z. Macosko. Slide-seq : A scalable technology for measuring genomewide expression at high spatial resolution. Science, 363(6434) :1463-1467, 2019.

[54] H. Safdari, A. Kalirad, C. Picioreanu, R. Tusserkani, B. Goliaei, and M. Sadeghi. Noise-driven cell differentiation and the emergence of spatiotemporal patterns. PLoS One, 15(4) :e0232060, 2020.

[55] A. Sarkar and M. Stephens. Separating measurement and expression models clarifies confusion in single cell RNA-seq analysis. bioRxiv, page 2020.04.07.030007, 2020.

[56] J.D. Scargle, J.P. Norris, B. Jackson, and J. Chiang. Studies in astronomical time series analysis. VI. Bayesian block representations. The Astrophysical Journal, 764(2) :167, feb 2013.

[57] G. Schiebinger, J. Shu, M. Tabaka, B. Cleary, V. Subramanian, A. Solomon, J. Gould, S. Liu, S. Lin, P. Berube, L. Lee, J. Chen, J. Brumbaugh, P. Rigollet, K. Hochedlinger, R. Jaenisch, A. Regev, and E. S. Lander. Optimal-transport analysis of single-cell gene expression identifies developmental trajectories in reprogramming. Cell, 176(4):928-943 e22, 2019.

[58] E. Schrödinger. What Is Life? The Physical Aspect of the Living Cell. Cambridge University Press, 1944.

[59] M. Setty, V. Kiseliovas, J. Levine, A. Gayoso, L. Mazutis, and D. Pe'er. Characterization of cell fate probabilities in single-cell data with Palantir. Nat Biotechnol, 37(4) :451-460, 2019.

[60] V. Shahrezaei and P. S. Swain. Analytical distributions for stochastic gene expression. Proc Natl Acad Sci U S A, 105(45) :17256-61, 2008.

[61] C.E. Shannon. A mathematical theory of communication. Bell System Technical Journal, 27 :379-423, 1948.

[62] X. Shao, X. Lu, J. Liao, H. Chen, and X. Fan. New avenues for systematically inferring cell-cell communication : through single-cell transcriptomics data. Protein Cell, 2020. 
[63] J. Shi, A. E. Teschendorff, W. Chen, L. Chen, and T. Li. Quantifying Waddington's epigenetic landscape : a comparison of single-cell potency measures. Brief Bioinform, page 20180040, 2018.

[64] K. Street, D. Risso, R. B. Fletcher, D. Das, J. Ngai, N. Yosef, E. Purdom, and S. Dudoit. Slingshot : cell lineage and pseudotime inference for single-cell transcriptomics. BMC Genomics, 19(1) :477, 2018.

[65] C. Strell, M. M. Hilscher, N. Laxman, J. Svedlund, C. Wu, C. Yokota, and M. Nilsson. Placing RNA in context and space - methods for spatially resolved transcriptomics. FEBS J, 286(8) :1468-1481, 2019.

[66] P.S. Stumpf, R.C.G. Smith, M. Lenz, A. Schuppert, F.-J. Müller, A Babtie, T.E. Chan, M.P.H. Stumpf, C.P. Please, S.D. Howison, F. Arai, and B.D. MacArthur. Stem cell differentiation as a non-markov stochastic process. Cell Systems, 5 :268-282, 2017.

[67] O. Symmons and A. Raj. What's luck got to do with it : Single cells, multiple fates, and biological nondeterminism. Mol Cell, 62 :788-802, 2016.

[68] A. E. Teschendorff and T. Enver. Single-cell entropy for accurate estimation of differentiation potency from a cell's transcriptome. Nat Commun, 8 :15599, 2017.

[69] G. Triqueneaux, C. Burny, O. Symmons, S. Janczarski, H. Gruffat, and G. Yvert. Cell-to-cell expression dispersion of B-cell surface proteins displays genetic variation among humans. Communications Biology. In press, 2020.

[70] E. Ventre, T. Espinasse, C.-E. Brehier, V. Calvez, T. Lepoutre, and O. Gandrillon. Reduction of a stochastic model of gene expression : Lagrangian dynamics gives acces to basins of attraction as cell types and metastability. bioRxiv, page 2020.09.04.283176, 2020.

[71] M. Villani, A. Barbieri, and R. Serra. A dynamical model of genetic networks for cell differentiation. PLoS One, 6(3) :e17703, 2011.

[72] K. Wallis. A note on the calculation of entropy from histograms. MPRA Paper, 2006.

[73] S. Wang, M. L. Drummond, C. F. Guerrero-Juarez, E. Tarapore, A. L. MacLean, A. R. Stabell, S. C. Wu, G. Gutierrez, B. T. That, C. A. Benavente, Q. Nie, and S. X. Atwood. Single cell transcriptomics of human epidermis identifies basal stem cell transition states. Nature Communications, 11(1) :4239, 2020.

[74] S. Wang, M. Karikomi, A. L. MacLean, and Q. Nie. Cell lineage and communication network inference via optimization for single-cell transcriptomics. Nucleic Acids Res, 47(11) :e66, 2019.

[75] S. Wang, J. Tu, L. Wang, and Z. Lu. Entropy-based model for miRNA isoform analysis. PLoS One, 10(3) :e0118856, 2015.

[76] K. Wiesner, J. Teles, M. Hartnor, and C. Peterson. Haematopoietic stem cells : entropic landscapes of differentiation. Interface Focus, 8(6) :20180040, 2018.

[77] Q. R. Xing, N. O. Cipta, K. Hamashima, Y. C. Liou, C. G. Koh, and Y. H. Loh. Unraveling heterogeneity in transcriptome and its regulation through single-cell multi-omics technologies. Front Genet, $11: 662,2020$.

[78] Y. Ye, Z. Yang, and J. Lei. Using single-cell entropy to describe the dynamics of reprogramming and differentiation of induced pluripotent stem cells. bioRxiv, page 2020.04.13.040311, 2020.

[79] X. Zheng, S. Jin, Q. Nie, and X. Zou. scRCMF : Identification of cell subpopulations and transition states from single-cell transcriptomes. IEEE Trans Biomed Eng, 67(5) :1418-1428, 2020. 